\title{
Coronary artery disease: arterial remodelling and clinical presentation
}

P C Smits, G Pasterkamp, M A Quarles van Ufford, F D Eefting, P R Stella, P P T de Jaegere, C Borst

\begin{abstract}
Objective-To investigate the hypothesis that in coronary artery disease large plaques in compensatorily enlarged segments are associated with acute coronary syndromes, whereas smaller plaques in shrunken segments are associated with stable angina pectoris.

Methods-Patients selected for percutaneous transluminal coronary angioplasty (PTCA) were divided into two groups, one with stable angina pectoris (stable group, $n=37$ ) and one with unstable angina or postmyocardial infarction angina of the infarct related artery (unstable group, $n=32$ ). In both groups, remodelling at the culprit lesion site was determined by intravascular ultrasound before the intervention. Remodelling was calculated as relative vessel area: [vessel area culprit lesion site $\div$ mean vessel area of both proximal and distal reference sites] $\times$ $100 \%$. Compensatory enlargement was defined as remodelling of $\geqslant 105 \%$, whereas shrinkage was defined as remodelling of $\leqslant 95 \%$.
\end{abstract}

Results-In the unstable group, the vessel area at the culprit lesion site was larger than in the stable group, at mean (SD) 18.1 (5.3) $v 14.6(5.4) \mathrm{mm}^{2}(\mathrm{p}=0.008)$. Lumen areas were similar. Consequently, plaque area and percentage remodelling were larger in the unstable group than in the stable group: mean (SD) 14.8 (4.8) $v$ $11.6(4.9) \mathrm{mm}^{2}(\mathrm{p}=0.009)$ and $112(31) \% v$ $95(17) \%(p=0.005)$, respectively. Significantly more culprit lesion sites were classified as shrunken in the stable group (21/ $37)$ than in the unstable group (8/32; $p=0.014)$. On the other hand, more lesion sites were classified as enlarged in the unstable group $(16 / 23)$ than in the stable group (8/37; $p=0.022)$.

Conclusions-In patients selected for PTCA, the mode of remodelling is related to clinical presentation.

(Heart 1999;82:461-464)

Keywords: atherosclerosis; coronary disease; remodelling; intravascular ultrasound

Dr P C Smits, Department of Interventional Cardiology,

Heart Center Rotterdam,

location Thoraxcenter,

University Hospital

Rotterdam, Dr

Molenwaterplein 40, 3015

GD Rotterdam, Netherlands.

email: smits@cardio.azr.nl

Accepted for publication 13 April 1999 and shrinkage have been observed with histology ${ }^{23}$ and in vivo with intravascular ultrasound. ${ }^{4-6}$ The relation between the mode compensatory enlargement, ${ }^{1}$ non-remodelling of coronary artery remodelling and its clinical presentation has not yet been investigated. In the femoral artery, we previously showed that significantly more histopathological markers for plaque vulnerability were present in compensatorily enlarged lesions with large plaques compared with shrinkage lesions with small plaques. $^{7}$ Because plaque vulnerability and inflammation are associated with plaque rupture, ${ }^{89}$ we postulated that lesions responsible for acute coronary syndromes consist predominantly of large plaques in enlarged vessel segments. We investigated this hypothesis by intravascular ultrasound in 69 patients who were selected for percutaneous transluminal coronary angioplasty (PTCA) of de novo atherosclerotic lesions.

\section{Methods}

Thirty consecutive patients were selected prospectively after a retrospective intravascular coronary ultrasound (ICUS) database study of 39 patients. Only patients with an angiographic focal lesion in the proximal or mid coronary artery segment studied by ICUS before a scheduled PTCA procedure were included. A focal lesion was defined as an angiographic lumen narrowing of at least $60 \%$ compared with normal looking adjacent segments, and a length of $\leqslant 20 \mathrm{~mm}$ by visual estimation. The culprit lesion was determined by angiography, thallium scintigraphy, stress echocardiography, or ECG changes during angina pectoris. Sixty nine de novo atherosclerotic lesions in native coronary arteries were studied. All patients had angina of class II-IV, classified according to the Canadian Cardiovascular Society criteria. In all cases the PTCA procedure was done electively.

Patients were divided into two groups on the basis of their clinical symptoms. The stable group consisted of patients with stable angina and no history of recent myocardial infarction. The unstable group consisted of patients with unstable angina or postmyocardial infarction angina. Only patients with unstable angina of class IIB, IIC, IIIB, or IIIC according to Braunwald's classification ${ }^{10}$ while receiving antiischaemic drug treatment were included in the unstable group. In the patients with unstable angina, PTCA of the culprit lesion was done at a median of 10 days (range 4 to 29) after admission to hospital; in the patients with postmyocardial infarction angina, PTCA of the culprit lesion in the infarct related artery was done at a median of 30 days (range 7 to 180 days) after the myocardial infarct. The characteristics of the patient groups are given in table 1 . 
Table 1 Characteristics of the patient population

\begin{tabular}{llll}
\hline & Stable group & Unstable group & p Value \\
\hline Total number of patients & 37 & 32 & \\
Male/female & $24 / 13$ & $26 / 6$ & 0.129 \\
Age (years) (mean (SD)) & $60(10)$ & $59(10)$ & 0.578 \\
Angina class (CCS) & & 9 & \\
$\quad$ Class II & 10 & 6 & \\
Class III & 27 & 17 & \\
Class IV & 0 & 15 & \\
$\quad$ Postinfarct angina & 0 & & \\
Vessel disease & & $28 / 4 / 0$ & 0.009 \\
Single/double/triple & $20 / 15 / 2$ & $18 / 13 / 1$ & 0.083 \\
Culprit lesion artery & $14 / 16 / 7$ & $17 / 31$ & 0.098 \\
RCA/LAD/Cx & $26 / 35$ & $20 / 29$ & 0.472 \\
Hypercholesterolaemia & $18 / 30$ & $19 / 27$ & 0.087 \\
Family history† & $16 / 33$ & $3 / 32$ & 0.526 \\
Smoking & $2 / 37$ & $6 / 30$ & 0.174 \\
Diabetes mellitus & $12 / 34$ & & \\
Hypertension & & $32 / 32$ & 1.000 \\
Drug treatment before ICUS & $36 / 37$ & $27 / 32$ & 0.947 \\
Aspirin & $31 / 37$ & $15 / 32$ & 0.938 \\
$\quad$ B Blocker & $17 / 37$ & $6 / 32$ & 0.008 \\
Calcium antagonist & $0 / 37$ & $4 / 32$ & 0.042 \\
Heparin (sc, iv) & $0 / 37$ & $20 / 32$ & 0.298 \\
Glyceryl trinitrate (iv) & $28 / 37$ & $13 / 32$ & 0.230 \\
Nitrates (oral) & $21 / 37$ & $3 / 32$ & 0.123 \\
Statins & $9 / 37$ & & \\
ACE inhibitors & & & \\
& & &
\end{tabular}

Values are numbers of patients unless otherwise stated. In some cases the data were unavailable. ${ }^{\star}$ Hypercholesterolaemia: total cholesterol $>5.4 \mathrm{mmol} / \mathrm{l}$; ffamily history: family history of coronary artery disease before 60 years of age.

ACE, angiotensin converting enzyme; CCS, Canadian Cardiovascular Society; Cx, circumflex coronary artery; ICUS, intracoronary ultrasound; iv, intravenous; LAD, left anterior descending coronary artery; RCA, right coronary artery; sc, subcutaneous.

INTRAVASCULAR ULTRASOUND SYSTEM AND IMAGING PROCEDURE

The intracoronary ultrasound procedures were performed with the Cardiovascular Imaging System (CVIS Inc, Sunnyvale, California, USA) coupled to a $2.9 \mathrm{~F}$ MicroView $30 \mathrm{MHz}$ coronary imaging catheter, or alternatively with the Endosonics System (Endosonics Corp, Rancho Cordova, California, USA) coupled to a $3.0 \mathrm{~F} 20 \mathrm{MHz}$ dynamic aperture array catheter with 32 (Oracle Megasonics, Endosonics Corp) or 64 (Visions Five-64, Endosonics Corp) echo elements. After coronary angiography, the culprit lesion was crossed with the imaging catheter over a 0.014 inch $(0.36 \mathrm{~mm})$ guide wire. Intravascular ultrasound images were obtained by a slow manual or motorised ( 0.5 to $1.0 \mathrm{~mm} / \mathrm{s})$ pull-back manoeuvre across the lesion and its adjacent segments. Glyceryl trinitrate was given intravenously during the procedure as an infusion drip ( $5 \mathrm{mg} / \mathrm{h}$ ) or as an intracoronary bolus infusion of $200 \mu \mathrm{g}$. The intravascular ultrasound images and voices of the physicians were recorded in parallel with the fluoroscopy on high resolution S-VHS videotapes for off-line analysis. To correlate the fluoroscopy and the intravascular ultrasound images, a time code was added to each signal by two synchronised video frame counters, or both video signals were mixed on-line into one signal and recorded.

\section{QUANTITATIVE INTRAVASCULAR ULTRASOUND} MEASUREMENTS

The intravascular ultrasound images were analysed off-line with a digital analyser, as described previously, ${ }^{11}$ by an independent observer (GP) who was unaware of the medical history of the patient. In each stenosed arterial segment, three sites were selected for quantitative intravascular ultrasound analysis. The site with the most severe lumen obliteration on the intravascular ultrasound images was selected, together with one proximal reference site and one distal reference site in each adjacent proximal and distal segment. The reference sites had no major side branches in between.

In the three selected cross sections, the lumen area $\left(\mathrm{mm}^{2}\right)$ and the area circumscribed by the external elastic lamina (vessel area, $\mathrm{mm}^{2}$ ) were traced. The external elastic lamina was defined as the outer border of the echolucent zone, which represents the interface between media and adventitia. ${ }^{12}$ The media plus atherosclerotic plaque area was defined as plaque area $\left(\mathrm{mm}^{2}\right)$ and calculated by subtracting the lumen area from the vessel area. To compare vessel area at the lesion with its reference area, we defined the reference vessel area as [vessel area proximal + vessel area distal]/2. When a proximal or distal side branch was present adjacent to the lesion, only the vessel area distal to or proximal to the lesion, respectively, was taken as a reference. Furthermore, only angiographically normal looking reference sites which showed less than $50 \%$ lumen obliteration on intravascular ultrasound were used for further calculations. Three proximal and five distal reference sites were excluded.

Arterial remodelling was calculated as relative vessel area: [lesion vessel area/reference vessel area] $\times 100 \%$. The lesions were divided into three groups based on the percentage relative vessel area: shrinkage (relative vessel area $\leqslant 95 \%$ ), non-remodelling (relative vessel area between $95 \%$ and $105 \%$ ), compensatory enlargement (relative vessel area $\geqslant 105 \%$ ). ${ }^{13}$

\section{STATISTICS}

All values are presented as mean (SD). Comparison of data was performed using a two tailed Student's $t$ test for continuous variables and a $\chi^{2}$ test for dichotomous and categorical variables, or Fisher's exact test when cell numbers were 5 or less. When the continuous variables were not parametrically distributed, a Mann-Whitney U test was performed. A p value less than 0.05 was considered statistically significant.

\section{Results}

Five patients were excluded at the time of the database analysis because of insufficient intravascular image quality caused by extensive calcification $(n=2)$ or double severe lesions in the culprit artery $(n=2)$, or because of more than $50 \%$ luminal stenosis by plaque in both proximal and distal reference sites $(\mathrm{n}=1)$. In all, 69 lesions in 69 patients were included in the study (39 of 44 patients retrospectively; 30 patients prospectively and consecutively).

The intravascular ultrasound measurements of the reference lumen and vessel areas were similar in the two groups (fig 1). At the lesion site, lumen area was similar in the two groups. However, the vessel area in the stable group was significantly smaller than in the unstable group, at $14.6 \quad(5.4)$ v $18.1 \quad(5.3) \mathrm{mm}^{2}$ $(p=0.008)$. Correspondingly, the plaque area was significantly smaller in the stable group than in the unstable group, at $11.6(4.9) v 14.8$ 

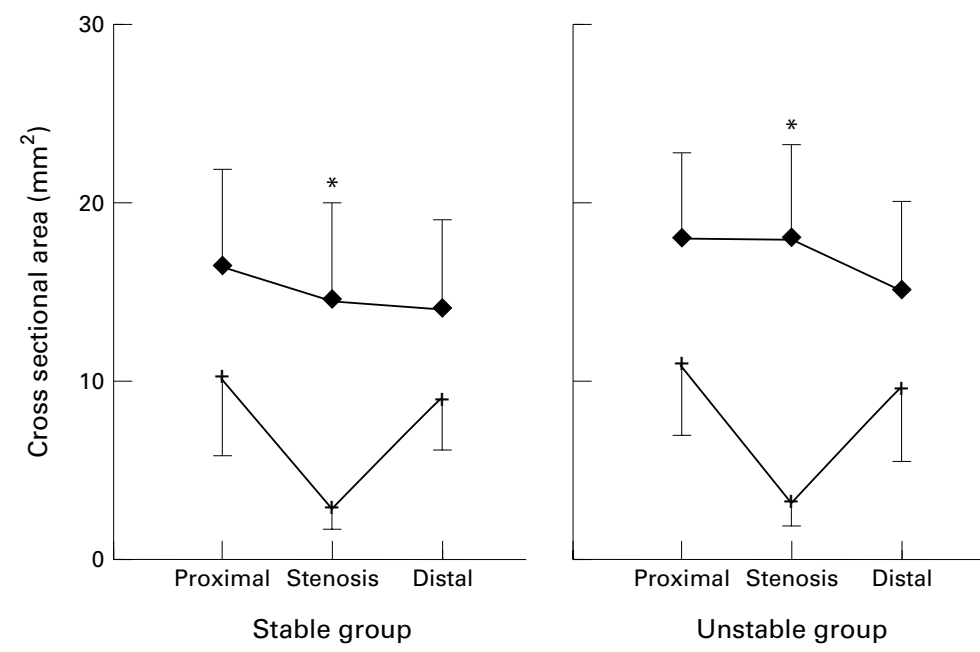

Figure 1 Mean cross sectional area values of lumen (+) and vessel ( ) at the proximal reference, stenosis, and distal reference sites in the two groups of patients. Error bars are standard deviations. Only vessel area at the stenosis site ( $\left.{ }^{*}\right)$ was significantly different between the groups: $14.6(5.4) v 18.1$ (5.3) $\mathrm{mm}^{2}(p=0.008)$.

Table 2 Relation between mode of coronary remodelling and symptoms of the culprit lesion

\begin{tabular}{lrlr}
\hline & Shrinkage & Non-remodelling & $\begin{array}{l}\text { Compensatory } \\
\text { enlargement }\end{array}$ \\
\hline Stable group & 21 & 8 & 8 \\
Unstable group & 8 & 8 & 16 \\
\hline
\end{tabular}

(4.8) $\mathrm{mm}^{2}(\mathrm{p}=0.009)$. In the stable group, percentage remodelling at the culprit lesion site was less than in the unstable group (95 (17)\% v $112(31) \% ; \mathrm{p}=0.005)$.

More shrunken culprit lesion sites (remodelling $\leqslant 95 \%$ ) were present in the stable group, whereas more compensatorily enlarged culprit lesions sites (remodelling $\geqslant 105 \%$ ) were found in the unstable group (table $2, \mathrm{p}=0.017$ ).

\section{Discussion}

In this intracoronary ultrasound study, we report a relation between the clinical symptoms of coronary artery disease and the form of remodelling at the culprit lesion site. Patients with stable angina selected for PTCA showed significantly less compensatory coronary enlargement at the culprit lesion site than patients with unstable angina or postmyocardial infarction angina. When the culprit lesion sites were arbitrarily divided in three different remodelling groups according to the percentage remodelling, ${ }^{13}$ then focal shrinkage was more prevalent in patients with stable angina, whereas focal compensatory enlargement was more prevalent in patients with unstable or postmyocardial infarction angina.

Little is known about the relation between remodelling mode and plaque vulnerability. Previously, we reported in a necropsy study of femoral arteries that histological markers for vulnerability of the atherosclerotic plaque were significantly more likely to be present in large plaques and enlarged vessel areas. ${ }^{7}$ Recently, Fishbein and Siegel ${ }^{14}$ postulated that not small but rather large plaques are more prone to fissuring and rupture, causing acute coronary syndromes as a result. Preliminary results from that group ${ }^{15}$ in 22 patients showed similar find- ings to ours in the present study. Furthermore, other investigators ${ }^{16}$ reported a larger mean vessel area and lesion area of culprit lesions in patients with unstable angina than in patients with stable angina.

The relation between larger plaque area at compensatorily enlarged lesion sites and acute coronary syndromes contrasts with previous serial angiographic studies. ${ }^{17}{ }^{18}$ From the latter it was inferred that plaque area, estimated from angiographic luminal narrowing, had little relation to the occurrence of $\mathrm{Q}$ wave myocardial infarction. In contrast to angiography, however, histopathological studies from patients with fatal coronary infarcts have consistently shown that at the site of plaque rupture the underlying lesion has caused severe lumen narrowing. ${ }^{19-21}$ Furthermore, in a study by Mann and Davies, ${ }^{22}$ histological measurements of coronary artery segments from patients who died suddenly from ischaemic heart disease revealed that the overall lumen obliteration is up to $30 \%$ greater than the angiographic measurements of the stenosis. These discrepancies can be explained by taking into account the different ways of measuring stenosis used in angiographic and pathological studies, as well as by the presence of arterial remodelling. ${ }^{23}$ With quantitative angiography, the stenosis is determined by comparing lumen diameter at the stenosis site with lumen diameter at an adjacent angiographically normal looking reference site. The histological method of measuring stenosis uses the diameter or cross sectional area of the vessel at the stenosis site as the reference value. Because both methods ignore the influence of atherosclerotic arterial remodelling, they are inadequate for investigating the relation between plaque area and remodelling and plaque vulnerability.

The risk of plaque disruption is related to intrinsic properties of the plaque. ${ }^{89}$ Histopathological studies by Kragel et al reported a linear increase in atheromatous core and inflammatory infiltrates and a linear decrease in cellular fibrous tissue with increasing cross sectional area narrowing in coronary artery lesions of patients who died with unstable angina. ${ }^{24} 25$ This implies that large plaques are likely to have more vulnerability properties than small plaques.

Although it is known that not all episodes of plaque disruption are followed by clinical sequelae, ${ }^{19} 26$ intravascular ultrasound assessment of coronary remodelling may be a powerful tool to determine which lesions are prone to fissuring or rupture. New prospective serial intravascular ultrasound studies are needed to confirm the validity of intravascular ultrasound for risk stratification of atherosclerotic coronary artery lesions.

\section{LIMITATIONS OF THE STUDY}

In the first place, we only studied patients who were recruited for PTCA. Whether the present results may be extrapolated to lesions of no, or minor, clinical significance remains to be determined. Second, in most cases the reference segments contained atherosclerotic plaque, and remodelling could have taken place 
at these sites. This could have influenced the remodelling calculations. The dimensions of the reference segments, however, were similar in all groups. Third, significantly more multivessel disease was observed in the stable group. This could mean that more advanced atherosclerotic disease was present in that group. Whether this was a confounding factor is as yet unknown. At present, we do not know if the mode of remodelling is time dependent. Fourth, during the time delay (up to six months) between myocardial infarction and ICUS of the culprit lesion in the unstable group, changes in plaque morphology could have occurred. However, most of these lesions were studied within one month and only three patients were studied between three and six months. Fifth, this study does not explain the observed relation between the mode of remodelling and clinical symptoms. It is conceivable that inflammation of the arterial wall with subsequent enzymatic matrix metalloproteinase activity not only induces destabilisation of the cap of the atherosclerotic plaque, but also induces breakdown of the collagenous arterial skeleton with subsequent arterial enlargement.

CONCLUSIONS

In patients selected for PTCA, large plaques at compensatorily enlarged lesion sites were more often associated with acute coronary events, in contrast to small plaques at shrunken lesion sites, which were more often related to stable angina. Thus the mode of arterial remodelling was related to the clinical symptoms. PCS was supported by the Dutch Heart Foundation, grant
NHS 94-115. GP is a fellow of the Catharijne Foundation, NHS $94-115$. GP is
Utrecht, Netherlands

1 Glagov S, Weisenberg E, Zarins CK, et al. Compensatory enlargement of human atherosclerotic coronary arteries. $N$ Engl F Med 1987;316:1371-5.

2 Pasterkamp G, Wensing PJW, Post MJ, et al. Paradoxical arterial wall shrinkage may contribute to luminal narrowing of human atherosclerotic femoral arteries. Circulation 1995;91:1444-9.

3 Pasterkamp G, Schoneveld AH, Wolferen van W, et al. The mpact of atherosclerotic arterial remodeling on percentage of luminal stenosis varies widely within the arterial system: a postmortem study. Arterioscler Thromb Vasc Biol 1997;17: 3057-63.

4 Nishioka T, Luo H, Eigler NL, et al. Contribution of inadequate compensatory enlargement to development of human coronary artery stenosis: an in vivo intravascular ultrasound study. $\mathcal{F}$ Am Coll Cardiol 1996;27:1571-6.

5 Mintz GS, Kent KM, Pichard AD, et al. Contribution of inadequate remodeling to the development of focal coronary artery stenoses. An intravascular ultrasound study. Cirary artery stenoses. An in
culation 1997;95:1791-8.
6 Smits PC, Bos L, Quarles van Ufford MA, et al. Shrinkage of human coronary arteries is an important determinant of de novo atherosclerotic luminal stenosis: an in vivo intravascular ultrasound study. Heart 1998;79:143-7.

7 Pasterkamp G, Schoneveld AH, Wal van der AC, et al. Relation of arterial geometry to luminal narrowing and histological markers for plaque vulnerability: the remodeling paradox. F Am Coll Cardiol 1998;32:655-62.

8 Wal van der AC, Becker AE, Loos van der CM, et al. Site of intimal rupture or erosion of thrombosed coronary atherosclerotic plaques is characterized by an inflammatory process irrespective of the dominant plaque morphology. Circuation 1994;89:36-44.

9 Moreno PR, Falk E, Palacios IF, et al. Macrophage infiltration in acute coronary syndromes. Implications for plaque rupture. Circulation 1994;90:775-8.

10 Braunwald E. Unstable angina: a classification. Circulation 1989;80:410-1.

11 Wenguang L, Gussenhoven WJ, Zhong Y, et al. Validation of quantitative analysis of intravascular ultrasound images. Int quantitative analysis of intravascul

12 Potkin BN, Bartorelli AL, Gessert JM, et al. Coronary artery imaging with intravascular high-frequency ultrasound. Circulation 1990;81:1575-85.

13 Pasterkamp G, Borst C, Gussenhoven EJ, et al. Remodeling of de novo atherosclerotic lesions in femoral arteries: impact on mechanism of balloon angioplasty. $7 \mathrm{Am}$ Coll Cardiol 1995;26:422-8.

14 Fishbein MC, Siegel RJ. How big are coronary atherosclerotic plaques that rupture? Circulation 1996;94:2662-6.

15 Nishioka T, Luo H, Nagai T, et al. Impact of coronary artery remodeling on clinical manifestations of patients with de novo coronary artery lesions [abstract]. F Am Coll Cardiol 1997;29(suppl A):125A

16 Kearney P, Erbel R, Rupprecht HJ, et al. Differences in the morphology of unstable and stable coronary lesions and their impact on the mechanisms of angioplasty. Eur Heart $f$ 1996;17:721-30.

17 Ambrose JA, Tannenbaum MA, Alexopoulos D, et al. Angiographic progression of coronary artery disease and the development of myocardial infarction. $7 \mathrm{Am}$ Coll Cardiol 1988;12:56-62.

18 Little WC, Constantinescu M, Applegate RJ, et al. Can coronary angiography predict the site of a subsequent myocardial infarction in patients with mild-to-moderate coronary artery disease. Circulation 1988;78:1157-66.

19 Falk E. Plaque rupture with severe pre-existing stenosis precipitating coronary thrombosis. Characteristics of coronary atherosclerotic plaques underlying fatal occlusive thrombi. Br Heart f 1983;50:127-34.

20 Horie T, Sekiguchi M, Hirosawa K. Coronary thrombosis in pathogenesis of acute myocardial infarction. Histopathological study of coronary arteries in 108 necropsied cases logical study of coronary arteries in 108 necrop

21 Qiao JH, Fishbein MC. The severity of coronary atherosclerosis at sites of plaque rupture with occlusive thrombosis. $\mathcal{F}$ Am Coll Cardiol 1991;17:1138-42.

22 Mann JM, Davies MJ. Assessment of the severity of coronary artery disease at postmortem examination. Are the measurements clinically valid? Br Heart $\mathcal{F} 1995 ; 74: 528-30$.

23 Pasterkamp G, Wensing PJW, Hillen B, et al. Impact of local atherosclerotic remodeling on the calculation of percent luminal narrowing. Am $\mathcal{F}$ Cardiol 1997;79:402-5.

24 Kragel AH, Reddy SG, Wittes JT, et al. Morphometric analysis of the atherosclerotic plaques in the four major epicardial coronary arteries in acute myocardial infarction and in sudden death. Circulation 1989;80:1747-56.

25 Kragel AH, Gertz DS, Roberts WC. Morphologic comparison of frequency and types of acute lesions in the major epicardial coronary arteries in unstable angina pectoris, sudden coronary death and acute myocardial infarction. $\mathcal{F}$ Am Coll Cardiol 1991;18:801-8.

26 Davies MJ, Bland JM, Hangartner JRW, et al. Factors influencing the presence or absence of acute coronary artery thrombi in sudden ischaemic death. Eur Heart 7 1989;10: 201-8. 